\title{
Le residenze nella cura dei pazienti psichiatrici lungoassistiti
}

\author{
The residential facilities in the care of long-term psychiatric patients
}

\author{
PAOLO MARTINI
}

\section{INTRODUZIONE}

Residenza è una parola chiave per la psichiatria. Dall'inizio della sua storia si è posto il dilemma: residenza-comunità o ospedale? $\mathrm{E}$, più vicino nel tempo, ospedale con servizi complementari, tra cui le residenze, oppure servizi territoriali, tra cui le residenze, alternativi all'ospedale? Residenza come rivendicazione sociale del diritto alla casa o come ambiente terapeutico e strumento sanitario di cura? La residenza è uno dei miti della psichiatria, situata com'è per utenti e operatori tra mondo esterno ed interno, tra spazio di oggettivazione e luogo di soggettività interpersonale, tra esigenze di cura, controllo sociale e bisogni di crescita, idealità ed integrazione individuale e sociale. Le residenze sono perciò un oggetto complesso. Il loro numero, le funzioni e i significati sono espressione, non soltanto del loro funzionamento intrinseco, ma anche delle inter-relazioni con gli altri 4 elementi fondamentali del Dipartimento Salute Mentale (DSM): la cultura del gruppo pluridisciplinare degli operatori, in particolare sulla relazione terapeutica e sulla funzione terapeutica unitaria del DSM; il centro di salute mentale e le attività territoriali; $i$ centri diurni e le attività riabilitative; il Servizio Psichiatrico di Diagnosi e Cura (SPDC) e le attività ospedaliere.

\section{CONTESTO STORICO}

Le residenze, come alloggi protetti nel territorio, hanno iniziato a diffondersi con i servizi complementari all'Ospedale Psichiatrico (OP) (OMS, 1968). Ma è dall'epoca della deospedalizzazione massiccia dagli OP che le residenze sono al centro dell'atten-

Indirizzo per la corrispondenza: Dr. P. Martini, Dipartimento Salute Mentale, via Guido Monaco 13, 52100 Arezzo.

Fax (+39) 0575.305.023. zione. Dal 1960 al 1985, i ricoverati in OP sono diminuiti del $78.2 \%$ in USA, del $56.4 \%$ in Italia e del $48.7 \%$ in Gran Bretagna (Jones, 1988). In USA, per ovviare alle carenze assistenziali, dal 1977 i finanziamenti federali sono stati assegnati soltanto a quei Community Mental Health Centers che offrissero programmi diurni e residenziali per i malati gravi e cronici (General Accounting Office, 1977). Dal 1978, il National Institute of Mental Health ha indicato un modello sistemico di servizi territoriali, tra cui le residenze, definito Community Support System. In Gran Bretagna, nel graduale spostamento delle risorse dagli $\mathrm{OP}$, sono stati previsti posti di residenzialità psichiatrica e servizi complementari di alloggio sociale forniti da enti locali e privati. In Italia, la riforma sanitaria non ha indicato quali strutture, oltre al SPDC ospedaliero, dovessero essere a disposizione dei servizi territoriali. Vi sono state polemiche sull'opportunità delle residenze; alcuni temendo il rischio di abbandono dei malati, altri il rischio di psichiatrizzazione e neoistituzionalizzazione. Le esperienze che comunque hanno fornito i migliori risultati sono state quelle che hanno implementato servizi territoriali forti, onnicomprensivi delle principali strutture, tra cui le residenze. Di questo modello di servizio ha tenuto conto il Progetto-Obiettivo Salute Mentale (PO) (DPR 7.4.94). L'indicazione che il DSM sia un unico servizio, costituito da un organico pluridisciplinare e da quattro elementi strutturali, tra cui le residenze, è un fatto che potrebbe caratterizzare l'assistenza psichiatrica. Purtroppo ha due gravi limiti: non lega il modello del DSM al superamento dell'OP e non ha un finanziamento reale.

\section{POSTI E RUOLO DELLE RESIDENZE NEL DSM}

Il $\mathrm{PO}$ prevede almeno un posto ogni 10.000 abitanti e residenze con non più di 20 posti letto «con 
livelli di protezione medico-psico-sociale differenziati per pazienti del residuo manicomiale e $\mathrm{i}$ cosiddetti nuovi cronici». Il primo PO dopo il 1978 verosimilmente ha voluto assolvere ad una funzione di codifica di principi e di indirizzo, lasciando alle Regioni il compito della programmazione. Queste dovranno indicare il numero dei posti letto in residenza, in relazione al numero totale dei posti previsti come necessari per una popolazione determinata. Teoricamente, il numero dei posti letto che dovrebbero essere disponibili per 100.000 abitanti di età superiore ai 15 anni oscilla da un minimo di 50 ad un massimo di 100 (De Salvia, 1987). Le previsioni di posti letto in residenze oscillano grandemente secondo che siano sanitarie o sociali, secondo il gradiente assistenziale fornito nelle 24 ore. Soprattutto i posti letto residenziali, con assistenza psichiatrica continuativa nelle 24 ore, sono in rapporto al numero dei posti letto in Ospedale Generale e in OP. Il nodo dell'OP si ripropone con forza in Italia, dove almeno 30.000 persone vi sono ancora dimenticate. Le esigenze di programmazione in questo caso si congiungono in pieno con le esigenze etiche e professionali della psichiatria, che non può non basarsi sulla presa in carico dei malati più gravi. Il superamento degli OP è necessario per prendersi cura dei ricoverati, ma anche per dare una risposta complessiva ed articolata ai bisogni dei malati gravi dell'intera popolazione. Le opportunità di alloggio in servizi sociali completano, ma non sostituiscono, una funzione che è essenziale per un DSM alternativo all'OP. Un'ulteriore considerazione è che la risposta ai bisogni di un'intera popolazione definita non può essere data soltanto in termini dei posti letto intra od extra -ospedalieri, ma dei posti nei vari centri territoriali e delle prestazioni differenziate nella continuità di intervento strategico del DSM. Questo funziona come un sistema, per cui il prodotto salute dipende da come lavorano ed interagiscono gli elementi sottostrutturali fondamentali del DSM. In questo processo, la cultura del DSM (Martini, 1993) gioca un ruolo cruciale perché residenze, centri diurni, ambulatori e servizi ospedalieri, sono strumenti operativi che acquistano un senso ed una funzione secondo l'utilizzo che ne viene fatto.

\section{LE RESIDENZE IN AREZZO}

Le residenze sanitarie sono risorse dell'intervento territoriale di comunità che si basa sul lavoro domi- ciliare e di rete sociale. Per cui le residenze personali e familiari sono il primo riferimento tra $i$ luoghi di intervento del DSM. Il tasso medio annuo di lungoassistiti è di 541/100.000 abitanti di età superiore ai 15 anni (Martini \& Domenici, 1992). Il 90\% di loro vive nel proprio domicilio ed è aiutato nel suo abituale ambiente di vita. Il $10 \%$ vive in residenze sanitarie extraospedaliere con programmi finalizzati a breve, medio e lungo termine. Le residenze del DSM forniscono assistenza psichiatrica residenziale non ospedaliera, secondo gradienti differenziati di protezione e di cura nelle 24 ore. Due residenze con assistenza continuativa nelle 24 ore, ognuna con 15 17 posti letto, ospitano per la quasi totalità ex-lungodegenti, ivi compresi quelli delle altre zone della provincia che non erano ancora stati dimessi alla chiusura dell'OP. Al 31.03.1994 risultavano 28 lungoassistiti e lungoospitati, di cui 3 nuovi cronici, cioè mai ricoverati in OP prima del 31.12.1981. Queste residenze sono organizzate secondo il modello dello hospital-hostel, fornendo prestazioni sanitarie di tipo ospedaliero, ma garantendo anche spazi di tipo domestico e comunitario. Le attività di riabilitazione si svolgono dentro e fuori le residenze, privilegiando quelle di gruppo, ma avendo cura dei programmi terapeutici individuali. Oltre che per progetti a lungo termine, le residenze possono essere utilizzate per programmi di breve, media durata per utenti che non abbiano trovato un'adeguata risposta nei precedenti interventi del DSM. Un medico ed un gruppo di infermieri sono assegnati funzionalmente a tempo pieno alle residenze, mentre terapisti della riabilitazione, assistenti sociali ed altre figure sono presenti in orari definiti. Il DSM ha la massima cura di garantire un'autonomia funzionale a queste strutture compatibilmente con il bisogno che esse non siano altrettanti servizi separati, ma strutture complementari di un unitario sistema terapeutico. Altre due residenze hanno un'assistenza minimale di personale psichiatrico fino a 12 ore diurne. Una, con ricettività massima di 17 posti letto, ospitava, al 31.03.1994, 17 dimessi dall'OP. È organizzata secondo un modello comunitario e fornisce gli stessi programmi riabilitativi, individuali e di gruppo, delle due precedenti. L'altra residenza è un gruppo-appartamenti, distribuiti su due edifici adiacenti. I 10 piccoli alloggi sono stati utilizzati dal 1980 per la dimissione di lungodegenti. Dal 1986, è stata introdotta una distinzione funzionale. Sette alloggi, per un massimo di 14 posti, sono rimasti per i dimessi con progetti a lungo termine. Tre alloggi, per un massimo di cinque posti, sono stati utilizzati per program- 
mi personalizzati a breve, medio termine. Al 31.03.1994, risultavano 12 dimessi e 3 lungoassistiti con programma breve-medio. Gli utenti non duplicati che hanno usufruito di quest'ultima risorsa sono stati 22 , alcuni per più volte. Tutti i programmi di riabilitazione si svolgono fuori dalle residenze, salvo quelli a carattere domestico e della cura di sé. Salvo due infermieri che si alternano, al mattino ed al pomeriggio, gli altri operatori intervengono sulla base dei programmi terapeutici; gli assistenti sociali svolgono un ruolo di supervisione delle residenze. L'ambiente terapeutico è molto familiare e gestito secondo modalità psicosociali, anche se si avvale del supporto medico. Cinque mini-alloggi nelle case popolari rappresentano il livello di residenzialità con minore supporto sanitario. Sono una prospettiva-ponte rispetto all'assegnazione personale di un alloggio popolare. Al 31.03.1994, gli utenti erano 7. I programmi terapeutico-riabilitativi sono quelli territoriali.

I posti letto ospedalieri del DSM sono 4 in uno specifico spazio psichiatrico e 3 nei reparti medici del presidio ospedaliero. Non si sono formati lungodegenti in ospedale. L'OP è stato formalmente chiuso per l'intera provincia di Arezzo il 1.4.94. Il DSM ha un coordinamento funzionale con il Servizio Sociale, che gestisce le RSA per non autosufficienti dell'USL, e con il Gruppo Operativo per l'handicap.

I tassi, elevati e stabili negli anni, di prevalenza un giorno e prevalenza annua, in particolare per le psicosi schizofreniche e affettive, indicano che il DSM mantiene un rapporto continuativo con una quota importante di popolazione portatrice della patologia più grave (Martini \& Domenici, 1992). Svolge così le funzioni equivalenti precedentemente assicurate dall'OP, ma fornisce anche una risposta in termini di popolazione, rendendo possibile un approccio epidemiologico e di comunità.

\section{CONCLUSIONI}

Le residenze, più ancora dei centri diurni, condizioneranno nei prossimi anni la qualità dei servizi offerti dai DSM. Il pendolo del tempo sembra favorire il ritorno (De Caro, 1948), insieme ai trattamenti biologici ospedalieri per gli acuti, di forme di gestione sussistenziale per i cronici. La sfida, che sembra giocarsi tutta sul terreno economico, è in realtà in gran parte culturale. L'implementazione e la direzione dello sviluppo delle attività residenziali nei nuovi Servizi dipenderà dalla cultura complessiva, tecnica ed organizzativa, degli operatori dei DSM. Questa si è dimostrata essere il cemento necessario per assemblare il sistema DSM ed anche il propellente indispensabile per mantenere nel territorio un'adeguata relazione terapeutica con una clientela non selezionata e rappresentativa di un'intera popolazione.

\section{BIBLIOGRAFIA}

De Caro D. (1948). L'ergoterapia negli Ospedali Psichiatrici ed i moderni orientamenti nel trattamento e nell'assistenza dei malati di mente. Il Lavoro Neuropsichiatrico 3, 1-23.

De Salvia D. (1987). Rapporto dal registro psichiatrico dei casi di Portogruaro. Rivista Sperimentale di Freniatria 111, Supplemento al fasc. 4, pp. 881-976.

General Accounting Office (1977). Returning the Mentally Disabled to the Community: Government Needs to Do More. Washington DC.

Jones K. (1988). Experience in Mental Health. Sage Publications: London.

Martini P. (1993). Aspetti culturali dei nuovi servizi di salute mentale per la comunità. In La Cultura dei Servizi (ed. A. Brignone), pp. 115-136. Edizioni ETS: Pisa.

Martini P. \& Domenici F. (1992). Assistenza psichiatrica e monitoraggio dei servizi. Il Registro dei casi di Arezzo 1987-1990. Epidemiologia e Psichiatria Sociale 1, 29-43.

Organisation Mondiale del la Santé (1968). Les Soins Psychiatriques Hospitaliers et la Readaptation des Malades Mentaux. Bureau Regional de l'Europe: Copenhagen. 


\section{L'APATIA.}

\section{DIMENSIONI}

\section{PSICOPATOLOGICHE E PRATICA CLINICA}

\section{a CURA dI PaOlo PancherI e MASSIMO BIONDI}

Un libro di 192 pagine. Lire 38.500

Da alcuni anni l'interesse per i sintomi cosiddetti negativi della schizofrenia è andato crescendo e non vi è clinico che non riconosca come tra $i$ sintomi negativi, il ruolo dominante sia giocato dalle alterazioni psicopatologiche descritte come anaffettività, appiattimento, ottundimento $\mathrm{e}$ appannamento affettivo o, meglio, apatia. Il problema dell'apatia è in realtà più complesso. Il sintomo apatia, infatti, sembra caratterizzare taluni periodi o momenti della vita "normale"; inoltre esso si.presenta con caratteri "transnosografici" anche in molti disturbi non schizofrenici e in particolare in alcuni disturbi di personalità e in gran parte dei disturbi dello spettro depressivo.

Quali sono dunque i confini semeiologici e l'importanza diagnostica del sintomo apatia? E, soprattutto, quali sono i suoi meccanismi patofisiologici e gli strumenti terapeutici a nostra disposizione per ridurne gli effetti negativi e talora devastanti?

Con i contributi (tra gli altri) di: A.C. Altamura,

F. Bogetto, C. Maggini, G. Muscettola,

L. Pavan, A. Pazzagli, L. Ravizza, G.C. Reda,

R. Rossi, V. Volterra.

\section{Pensiero Scientifico Editore}

Libre e riviste, nostri contemporanei 